\title{
The emergent dynamical symmetry at the triple point of nuclear deformations
}

\author{
Yu Zhang, ${ }^{1}$ Feng Pan, ${ }^{1,2}$ Yu-xin Liu,,${ }^{3,4,5}$ Yan-An Luo, ${ }^{6}$ and J. P. Draayer ${ }^{2}$ \\ ${ }^{1}$ Department of Physics, Liaoning Normal University, Dalian 116029, P. R. China \\ ${ }^{2}$ Department of Physics and Astronomy, Louisiana State University, Baton Rouge, LA 70803-4001, USA \\ ${ }^{3}$ Department of Physics and State Key Laboratory of Nuclear Physics and Technology, Peking University, Beijing 100871, P. R. China \\ ${ }^{4}$ Collaborative Innovation Center of Quantum Matter, Beijing 100871, China \\ ${ }^{5}$ Center for High Energy Physics, Peking University, Beijing 100871, China \\ ${ }^{6}$ School of Physics, Nankai University, Tianjin 300071, P. R. China
}

(Dated: July 5, 2019)

\begin{abstract}
Based on the boson realization of the Euclidean algebras, it is shown that the five-dimensional Euclidean dynamical symmetry may emerge at the triple point of the shape phase diagram of the interacting boson model, which thus offers a symmetry-based understanding of this isolated point. It is further shown that the low-lying dynamics in ${ }^{108} \mathrm{Pd},{ }^{134} \mathrm{Ba},{ }^{64} \mathrm{Zn}$, and ${ }^{114} \mathrm{Cd}$ may be dominated by the Euclidean dynamical symmetry.

PACS numbers: 21.60.Fw, 21.60.Ev, 21.10.Re, 27.60.+j
\end{abstract}

\section{INTRODUCTION}

Dynamical symmetries (DSs) provide considerable insight into the nature of quantum many-body dynamical structures. Generally, DS occurs when the Hamiltonian of a system can be written in terms of Casimir operators of a chain of Lie algebras $G \supset G^{\prime} \supset G^{\prime \prime} \cdots$. Typical examples of DS are those associated with the interacting boson model (IBM) [1] for nuclear structure and the vibron model (VM) [2] for molecules and atomic clusters [3].

The IBM possesses an overall U(6) symmetry with three DSs corresponding to three typical collective structures or quadrupole deformations [2]; namely, a spherical vibrator $[\mathrm{U}(5)]$, an axially symmetric prolate rotor [SU(3)], and a $\gamma$ soft rotor $[\mathrm{O}(6)]$. In addition, an axially symmetric oblate rotor $[\overline{\mathrm{SU}(3)}]$ can be involved in the IBM dynamics if adopting an alternative $\mathrm{SU}(3)$ quandrupole operator in contrast to that often used for the prolate rotor [4]. Besides these exact DSs, the partial dynamical symmetries (PDS) [5-7] and quasidynamical symmetries (QDS) [8-10] have also been found to occur in the IBM. Indeed, it was found that the SU(3) QDS [11, 12] may emerge along the trajectory in the IBM parameter space close to the Alhassid-Whelan arc of regularity [13], which has been empirically confirmed [14]. A link between PDS and QDS has also been established recently via the method of quantum number fluctuation [15]. As there is a link between each DS in the IBM and the quantum (shape) phase or quadrupole deformation [1], the (shape) phase transitions in nuclei may be characterized as the quantum phase transitions (QPTs) in between the different DSs in the IBM [16-18]. Particularly, the QPT from SU(3) to $\overline{\mathrm{SU}(3)}$ may exactly occur at the point of O(6) DS [4]. In addition, an isolate triple point, at which three kinds of quadrupole deformations including the spherical, prolate, and oblate shapes may coexist at the same time [19,20], also emerges in the critical region of the IBM. On the other hand, an algebraic model of the Euclidean dynamical symmetry in 5-dimension $(\mathrm{Eu}(5)$ DS) [21] has recently been suggested to describe the nuclei in the critical region. Especially, it was shown [21] that the re- sults obtained from the simplest version of the Eu(5) DS (unprojected) in the large- $N$ limit are the same as those from the $\mathrm{E}(5)$ critical point symmetry (E(5) CPS) built from the Bohr Hamiltonian with an infinite well potential [22]. However, the relation between the IBM and the Eu(5) DS still remains to be revealed. In this work, we will present an extensive analysis of the $\mathrm{Eu}(5) \mathrm{DS}$ and clarify the relation between the $\mathrm{Eu}(5) \mathrm{DS}$ and the IBM.

\section{THE BOSON REALIZATION OF THE EUCLIDEAN ALGEBRA}

A Hamiltonian in the IBM framework is constructed from two kinds of boson operators; namely, a s-boson with $J^{\pi}=0^{+}$ and a $d$-boson with $J^{\pi}=2^{+}[1]$. The three DSs in the IBM are characterized by three different chains of the U(6) group [1]:

$$
\begin{aligned}
& \mathrm{U}(6) \supset \mathrm{U}(5) \supset \mathrm{SO}(5) \supset \mathrm{SO}(3), \\
& \mathrm{U}(6) \supset \mathrm{O}(6) \supset \mathrm{SO}(5) \supset \mathrm{SO}(3), \\
& \mathrm{U}(6) \supset \mathrm{SU}(3) \supset \mathrm{SO}(3)
\end{aligned}
$$

Then, the Hamiltonian with an explicit DS in the IBM can be written in terms of the Casimir operators of the corresponding group chain. On the other hand, it was shown [21] that one can use the $d$-boson operator to construct the Casimir operator of the $\mathrm{Eu}(5)$ group as

$$
\hat{C}_{2}[\operatorname{Eu}(5)]=\hat{n}_{d}+\frac{5}{2}-\frac{1}{2}\left(\hat{P}_{d}^{\dagger}+\hat{P}_{d}\right),
$$

where $\hat{n}_{d}=\sum_{u} d_{u}^{\dagger} d_{u}$, and $\hat{P}_{d}=\sum_{u}(-)^{u} d_{u} d_{-u}$. Accordingly, the $d$-boson operator can be also used to construct the fifteen generators of the $\mathrm{Eu}(5)$ Lie algebra as

$$
\begin{aligned}
& \hat{Q}_{u}^{(2)}=\frac{1}{\sqrt{2}}\left[\tilde{d}_{u}-d_{u}^{\dagger}\right], \\
& \hat{T}_{u}^{(\lambda)}=\sqrt{2}\left(d^{\dagger} \tilde{d}\right)_{u}^{(\lambda)}, \lambda=1,3,
\end{aligned}
$$

where $\tilde{d}_{u}=(-1)^{u} d_{u}$. It is evident that the Eu(5) algebra is non-compact as seen from (3). It should be mentioned that 
a geometric realization of the $\mathrm{Eu}(5)$ algebra in the collective model may be constructed by using the quadrupole coordinates $q_{u}$ and the conjugate momenta $\tilde{p}_{u}[23]$. The boson algebraic and the collective geometric realizations may be linked by implementing the $d$-boson operator with $\tilde{d}_{u}=\frac{1}{\sqrt{2}}\left[q_{u}+i \tilde{p}_{u}\right]$ and $d_{u}^{\dagger}=\frac{1}{\sqrt{2}}\left[q_{u}-i \tilde{p}_{u}\right][21]$.

It can be proven that the above $\mathrm{Eu}(5)$ generators satisfy the commutation relations

$$
\begin{aligned}
& {\left[\hat{Q}_{u}^{(2)}, \hat{Q}_{v}^{(2)}\right]=} 0, \\
& {\left[\hat{T}_{u}^{(\lambda)}, \hat{Q}_{v}^{(2)}\right]=}-\sqrt{\frac{4 \lambda+2}{5}}\langle\lambda u 2 v \mid 2 u+v\rangle \hat{Q}_{u+v}^{(2)}, \\
& {\left[\hat{T}_{u}^{(\lambda)}, \hat{T}_{u^{\prime}}^{\left(\lambda^{\prime}\right)}\right]=-\sqrt{8(2 \lambda+1)\left(2 \lambda^{\prime}+1\right)} \sum_{k=\text { odd }}\left\{\begin{array}{c}
\lambda, \lambda^{\prime}, k \\
2,2,2
\end{array}\right\} \times } \\
& \quad\left\langle\lambda u \lambda^{\prime} u^{\prime} \mid k u+u^{\prime}\right\rangle \hat{T}_{u+u^{\prime}}^{(k)},
\end{aligned}
$$

and

$$
\left[\hat{Q}_{u}^{(2)}, \hat{C}_{2}[\operatorname{Eu}(5)]\right]=\left[\hat{T}_{u}^{(\lambda)}, \hat{C}_{2}[\operatorname{Eu}(5)]\right]=0 .
$$

One can further prove that the operators $\left\{\hat{T}_{u}^{(\lambda)}\right\}$ with $\lambda=1,3$ generate the $\mathrm{SO}(5)$ algebra, in which the angular momentum operators defined by $\left\{\hat{L}_{u}=\sqrt{5} \hat{T}_{u}^{(1)}\right\}$ generate the $\mathrm{SO}(3)$ algebra. The $\mathrm{Eu}(5)$ algebra may be characterized by the algebraic chain [23]

$$
\mathrm{Eu}(5) \supset \mathrm{SO}(5) \supset \mathrm{SO}(3)
$$

In addition, $\left\{\hat{Q}_{u}^{(2)}\right\}$ generate the Abelian group $\mathrm{T}_{5}$ of translations in the five dimensional space. It is thus realized that the $\mathrm{Eu}(5)$ algebra is equivalent to the semidirect sum of $\mathrm{T}_{5}$ and $\mathrm{SO}(5)$, namely, $\mathrm{Eu}(5)=\mathrm{T}_{5} \oplus_{S} \mathrm{SO}(5)$ [24 -26$]$. Accordingly, there is another dynamical symmetry related with the algebraic chain

$$
\mathrm{Eu}(5) \supset \mathrm{T}_{5} \oplus_{s} \mathrm{SO}(3) \supset \mathrm{SO}(3),
$$

in which the semidirect sum $\mathrm{T}_{5} \oplus_{s} \mathrm{SO}(3)$ is often used to denote the dynamical symmetry of a quadrupole-deformed rigid body [27]. In the following, only the dynamical situation characterized by the algebraic chain (6) will be studied.

\section{THE SU(1,1) EXPRESSION OF THE EU(5) DS AND ITS LINK WITH THE E(5) CPS}

As analyzed in [21], the spectral structure of (2) in the large- $N$ limit coincides with that generated from the $\mathrm{E}(5)$ CPS [22]. Actually, the form of the Eu(5) Casimir operator shown in (2) can be directly translated from the Hamiltonian of the E(5) CPS via the SU $(1,1)$ reformulation. Specifically, the $\mathrm{SU}(1,1)$ Lie algebra generated by $\hat{S}_{v}, v=0, \pm$, satisfy the commutation relations [28-31]

$$
\left[\hat{S}_{-}, \hat{S}_{+}\right]=2 \hat{S}_{0}, \quad\left[\hat{S}_{0}, \hat{S}_{ \pm}\right]= \pm \hat{S}_{ \pm} .
$$

The Casimir operator of $\mathrm{SU}(1,1)$ can be written as

$$
\hat{C}_{2}[\mathrm{SU}(1,1)]=\hat{S}_{0}\left(\hat{S}_{0}-1\right)-\hat{S}_{+} \hat{S}_{-} .
$$

Let $|\lambda, k\rangle ; k=0,1,2, \cdots$ be the basis vectors of the irreducible representation $\lambda$ of $\mathrm{SU}(1,1)$, of which the matrix representation is determined by [28-31]

$$
\begin{aligned}
& \hat{S}_{0}|\lambda, k\rangle=\frac{1}{2}(\lambda+2 k)|\lambda, k\rangle, \\
& \hat{S}_{+}|\lambda, k\rangle=\sqrt{(\lambda+k)(k+1)}|\lambda, k+1\rangle, \\
& \hat{S}_{-}|\lambda, k\rangle=\sqrt{(\lambda+k-1) k}|\lambda, k-1\rangle, \\
& \hat{C}_{2}[\mathrm{SU}(1,1)]|\lambda, k\rangle=\frac{\lambda}{2}\left(\frac{\lambda}{2}-1\right)|\lambda, k\rangle .
\end{aligned}
$$

For the $d$-boson realization of the $\mathrm{SU}(1,1)$ [32],

$$
\hat{S}_{+}^{d}=\frac{1}{2} \hat{P}_{d}^{+}, \quad \hat{S}_{-}^{d}=\frac{1}{2} \hat{P}_{d}, \hat{S}_{0}^{d}=\frac{1}{2}\left(\hat{n}_{d}+\frac{5}{2}\right),
$$

from which the quantum numbers $\lambda$ and $k$ can be expressed by the seniority quantum number $\tau$ of the $\mathrm{SO}(5)$ and the number of the $d$-bosons $n_{d}$ as $\lambda=\tau+\frac{5}{2}$ and $k=\frac{1}{2}\left(n_{d}-\tau\right)$. Then, the Casimir operator of $\mathrm{Eu}(5)$ defined in (2) can be expressed as

$$
\hat{C}_{2}[\operatorname{Eu}(5)]=2 \hat{S}_{0}^{d}-\left(\hat{S}_{+}^{d}+\hat{S}_{-}^{d}\right) .
$$

Thus, one may choose to solve the eigenvalue problem of $\hat{C}_{2}[\operatorname{Eu}(5)]$ within the subspace spanned by the $\mathrm{SU}(1,1)$ basis vectors $\{|\lambda, k\rangle\}$. In addition, the $\mathrm{Eu}(5)$ with the Casimir operator given in (15) can easily be extended to the n-dimensional case. Specifically, the Casimir operator of the $\mathrm{n}$-dimensional Euclidean group $(\mathrm{Eu}(\mathrm{n}))$ with $n=2 l+1$ may be written as

$$
\hat{C}_{2}[\operatorname{Eu}(n)]=2 \hat{S}_{0}^{l}-\left(\hat{S}_{+}^{l}+\hat{S}_{-}^{l}\right),
$$

of which the $\mathrm{SU}(1,1)$ algebra is generated by [33]

$$
\begin{aligned}
& \hat{S}_{+}^{l}=\frac{1}{2} \hat{P}_{l}^{\dagger}, \quad \hat{S}_{-}=\frac{1}{2} \hat{P}_{l} \\
& \hat{S}_{0}^{l}=\frac{1}{2}\left(\hat{n}_{l}+\frac{2 l+1}{2}\right),
\end{aligned}
$$

where $\hat{n}_{l}=\sum_{m} l_{m}^{\dagger} l_{m}$, and $\hat{P}_{l}=\sum_{m}(-1)^{m} l_{m} l_{-m}$, in which $l_{m}^{\dagger}\left(l_{m}\right)$ are the creation (annihilation) operators of the $l$-bosons. It is obvious that the $\mathrm{SU}(1,1)$ algebra given in (14) is only a special case of (17) with $l=2$. Accordingly, one can also construct the $\mathrm{Eu}(\mathrm{n})$ algebra with the $l$-boson operators as

$$
\begin{aligned}
& \hat{Q}_{u}^{(l)}=\frac{1}{\sqrt{2}}\left[\tilde{l}_{u}-l_{u}^{\dagger}\right], \\
& \hat{T}_{u}^{(\lambda)}=\sqrt{2}\left(l^{\dagger} \tilde{l}\right)_{u}^{(\lambda)}, \lambda=1,3, \ldots, 2 l-1,
\end{aligned}
$$

of which the $l=2$ case just corresponds to the $\mathrm{Eu}(5)$ algebra given in (3).

On the other hand, the collective Bohr Hamiltonian of the E(5) CPS [22] is written as

$$
\begin{array}{r}
H_{E(5)}=-\frac{\hbar^{2}}{2 B}\left\{\frac{1}{\beta^{4}} \frac{\partial}{\partial \beta} \beta^{4} \frac{\partial}{\partial \beta}+\frac{1}{\beta^{2}}\left(\frac{1}{\sin 3 \gamma} \frac{\partial}{\partial \gamma} \sin 3 \gamma \frac{\partial}{\partial \gamma}\right.\right. \\
\left.\left.-\frac{1}{4} \sum_{k} \frac{L_{k}^{\prime 2}}{\left[\sin \left(\gamma-\frac{2}{3} n \pi\right)\right]^{2}}\right)\right\}+V(\beta)
\end{array}
$$


with

$$
V(\beta)= \begin{cases}0, & \beta \leq \beta_{W} \\ \infty, & \beta>\beta_{W}\end{cases}
$$

By writing the eigenfuctions

$$
\Phi(\beta, \gamma, \theta)=f(\beta) \Psi(\gamma, \theta),
$$

one can get the angular equation

$$
\begin{aligned}
& {\left[-\frac{1}{\sin 3 \gamma} \frac{\partial}{\partial \gamma} \sin 3 \gamma \frac{\partial}{\partial \gamma}+\frac{1}{4} \sum_{k} \frac{L_{k}^{\prime 2}}{\left[\sin \left(\gamma-\frac{2 k \pi}{3}\right)\right]^{2}}\right] \Psi(\gamma, \theta) } \\
= & \Lambda \Psi(\gamma, \theta),
\end{aligned}
$$

with $\Lambda=\tau(\tau+3)$ and the radial equation

$$
\left[-\frac{\hbar^{2}}{2 B}\left(\frac{1}{\beta^{4}} \frac{\partial}{\partial \beta} \beta^{4} \frac{\partial}{\partial \beta}-\frac{\Lambda}{\beta^{2}}\right)+V(\beta)\right] f(\beta)=E f(\beta) .
$$

According to the analysis shown in $[28-31]$, the $\mathrm{SU}(1,1)$ algebra can be alternatively defined in terms of the differential operators as

$$
\begin{gathered}
\hat{S}_{ \pm}^{\beta}=\frac{1}{4}\left[\frac{\triangle^{2}}{a^{2}}-\frac{\Lambda}{(a \beta)^{2}}+(a \beta)^{2} \mp\left(2 \beta \frac{\partial}{\partial \beta}+5\right)\right], \\
\hat{S}_{0}^{\beta}=\frac{1}{4}\left[-\frac{\triangle^{2}}{a^{2}}+\frac{\Lambda}{(a \beta)^{2}}+(a \beta)^{2}\right],
\end{gathered}
$$

with $\triangle^{2}=\frac{1}{\beta^{4}} \frac{\partial}{\partial \beta} \beta^{4} \frac{\partial}{\partial \beta}$ and $\frac{1}{a^{2}}=\frac{\hbar^{2}}{2 B}$. Then, the Hamiltonian associated with (23) in the infinite well can be written as

$$
H=2 \hat{S}_{0}^{\beta}-\left(\hat{S}_{+}^{\beta}+\hat{S}_{-}^{\beta}\right),
$$

which is the same form as that shown in (15). This indicates that the $\mathrm{Eu}(5) \mathrm{DS}$ can be directly translated from the $\mathrm{E}(5)$ CPS at the Hamiltonian level. Thus, solving the differential equation (23) is approximately equivalent to diagonalizing the Hamiltonian 260 within the subspace spanned by the basis vectors of $\mathrm{SU}(1,1)[28-31]$. Though it is not easy to translate the boundary conditions of the infinite well into those in the algebraic description, it can approximately be realized in the diagonalization of (15) with a cut-off in the Hilbert space [21].

\section{THE APPROXIMATE EU(5) DS AT THE TRIPLE POINT IN THE IBM}

Because the main composition of the system is the same as that of the IBM with $d$-bosons at the second quantization level, to investigate the relation between the IBM and the $\mathrm{Eu}(5) \mathrm{DS}$ at the Hamiltonian level, we consider the IBM consistent- $Q$ Hamiltonian [34]

$$
\hat{H}(\eta, \chi)=\varepsilon\left[(1-\eta) \hat{n}_{d}-\frac{\eta}{4 N} \hat{Q}^{\chi} \cdot \hat{Q}^{\chi}\right],
$$

where $\hat{Q}^{\chi}=\left(d^{\dagger} s+s^{\dagger} \tilde{d}\right)^{(2)}+\chi\left(d^{\dagger} \tilde{d}\right)^{(2)}$ is the quadrupole operator, $\eta$ and $\chi$ are the control parameters with $\eta \in[0,1]$ and $\chi \in[-\sqrt{7} / 2, \sqrt{7} / 2]$, and $\varepsilon$ is a scale factor. It can be proven that the Hamiltonian is in the U(5) DS when $\eta=0$; it is in the $\mathrm{O}(6) \mathrm{DS}$ when $\eta=1$ and $\chi=0$; it is in the SU(3) DS when $\eta=1$ and $\chi=-\frac{\sqrt{7}}{2}$; and it is in the $\overline{\mathrm{SU}(3)} \mathrm{DS}$ when $\eta=1$ and $\chi=\frac{\sqrt{7}}{2}$. The two-dimensional parameter space of 27) can be mapped onto a symmetric triangle (see Fig. 1), called the extended Casten triangle [4]. To identify the QPTs in the IBM, one may use the coherent state defined as [1]

$$
\begin{aligned}
|\beta, \gamma, N\rangle & =\frac{1}{\sqrt{N !\left(1+\beta^{2}\right)^{N}}}\left[s^{\dagger}+\beta \cos \gamma d_{0}^{\dagger}\right. \\
& \left.+\frac{1}{\sqrt{2}} \beta \sin \gamma\left(d_{2}^{\dagger}+d_{-2}^{\dagger}\right)\right]^{N}|0\rangle
\end{aligned}
$$

to obtain the scaled potential surface corresponding to the Hamiltonian (27) in the large- $N$ limit, which is given as

$$
\begin{aligned}
V_{\mathrm{s}}(\beta, \gamma) & =\left.\frac{1}{\varepsilon N}\langle\beta, \gamma, N|H| \beta, \gamma, N\rangle\right|_{N \rightarrow \infty} \\
& =(1-\eta) \frac{\beta^{2}}{1+\beta^{2}}-\frac{\eta}{4\left(1+\beta^{2}\right)^{2}} \times \\
& {\left[4 \beta^{2}-4 \sqrt{\frac{2}{7}} \chi \beta^{3} \cos 3 \gamma+\frac{2}{7} \chi^{2} \beta^{4}\right] . }
\end{aligned}
$$

To illustrate the type and the order of the QPTs, one should minimize the potential function 29 by varying $\beta$ and $\gamma$ for $\eta$ and $\chi$. The optimal values are denoted as $\beta_{\mathrm{e}}$ and $\gamma_{\mathrm{e}}$, from which one can get the ground state energy per boson defined as $E_{g}=V_{s}\left(\eta, \chi, \beta_{\mathrm{e}}, \gamma_{\mathrm{e}}\right)$. It can be found that the $\gamma$-dependence in (29) yields either $\gamma_{\mathrm{e}}=0^{\circ}$ or $\gamma_{\mathrm{e}}=60^{\circ}$, of which the case with $\gamma_{\mathrm{e}}=60^{\circ}$ can be equivalently described by substituting $\gamma_{\mathrm{e}}=0^{\circ}$ and $\beta=-\beta_{\mathrm{e}}$. Therefore, $\beta_{e}$ may serve as the order parameter [35] to identify the order and the type of the QPTs. For the second-order QPT, the order parameter $\beta_{\mathrm{e}}$ changes continuously, but with a discontinuous in the second derivative of 29). In contrast, the first-order QPT may involve a discontinuous jump in the order parameter $\beta_{\mathrm{e}}$ itself [35]. In addition, $\beta_{\mathrm{e}}=0, \beta_{\mathrm{e}}>0$ and $\beta_{\mathrm{e}}<0$ represent the spherical, prolate, and oblate deformations, respectively. Based on the criteria mentioned above, one can prove that the system may experience the first-order QPTs in two directions with changing of the control parameters $\eta$ and $\chi$ [19]. Specifically, the critical points of the first-order QPTs occurring in the $\eta$ direction are given as $\eta_{c}=\frac{14}{28+\chi^{2}}$ and $\chi \in[-\sqrt{7} / 2, \sqrt{7} / 2]$, and in the $\chi$ direction are characterized by $\chi_{c}=0$ with $\eta \in(0.5,1]$. Particularly, the crossing point of the first-order QPTs occurring in the two directions, namely $(\eta=0.5, \chi=0)$, may be recognized as the single triple point as it is the junction point of the spherical, prolate, and oblate deformations [19]. Meanwhile, this point is also proven to be the critical point of the second-order phase transition in the $\eta$ direction. The whole shape phase diagram corresponding to 29 is clearly shown in Fig.11. 


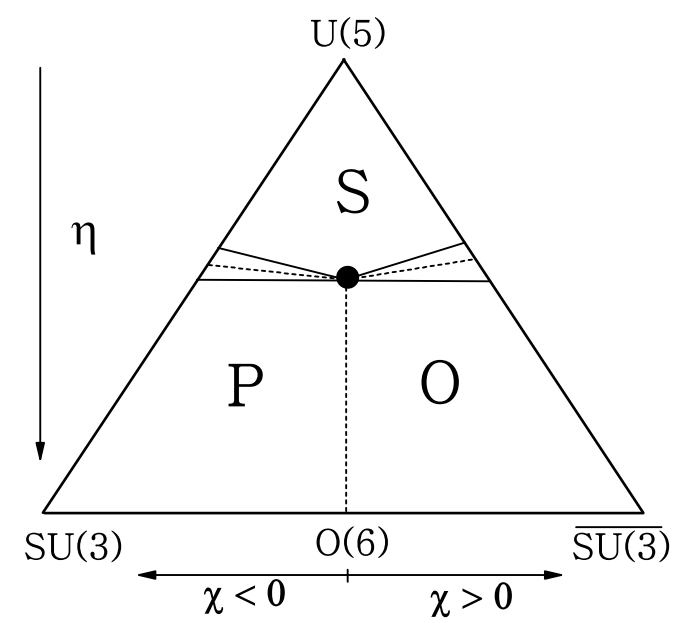

FIG. 1: (Color online) Shape phase diagram in the IBM parameter space, where $S$ represents the region with $\beta_{\mathrm{e}}=0$ corresponding to the spherical, $\mathrm{P}$ represents the region with $\beta_{\mathrm{e}}>0$ corresponding to the prolate, and $\mathrm{O}$ represents the region with $\beta_{\mathrm{e}}<0$ corresponding to the oblate. In addition, the dashed lines correspond to the critical points of the first-order QPTs, the two thin regions involving the dashed lines represent the two-phase coexisting regions [36], and the solid dot in the center represents the triple point.

As is known, if a system has an underlying symmetry of the group G, the corresponding Hamiltonian should commutes with the generators of the group G. Along this line, it has been proven [12] that there exists a parametrization trajectory preserving the approximate SU(3) symmetry (SU(3) QDS) inside the symmetry triangle of the IBM in the the large- $N$ limit. To identify the underlying Eu(5) DS in the IBM parameter space, we examine the commutation relations between the generators of the $\mathrm{Eu}(5)$ defined in (3) and the IBM Hamiltonian $\hat{H}(\eta, \chi)$ given in 27]. Firstly, it is easy to know that the IBM Hamiltonian does commute with the angular momentum operators $\hat{L}_{u}=\sqrt{5} \hat{T}_{u}^{(1)}$ since the Hamiltonian is a scalar. As a result, one only needs to examine the conditions under which the Hamiltonian may commute (approximately) with the other generators of the $\mathrm{Eu}(5)$. Specifically, one can prove the following commutation relations by using the standard angular momentum coupling techniques [12, 37]:

$$
\begin{aligned}
& {\left[\hat{T}_{u}^{(3)}, \hat{n}_{d}\right]=0,} \\
& {\left[\hat{T}_{u}^{(3)},\left(d^{\dagger} s+s^{\dagger} \tilde{d}\right)_{v}^{(2)}\right]} \\
& =-\frac{\sqrt{14}}{5}\langle 3 u 2 v \mid 2 u+v\rangle\left(d^{\dagger} s+s^{\dagger} \tilde{d}\right)_{u+v}^{(2)}, \\
& {\left[\hat{T}_{u}^{(3)},\left(d^{\dagger} \tilde{d}\right)_{v}^{(2)}\right]}
\end{aligned}
$$

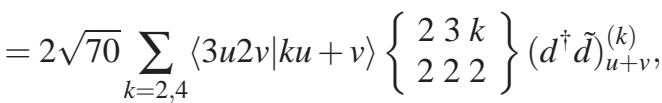

$$
\begin{aligned}
& {\left[\hat{Q}_{u}^{(2)}, \hat{n}_{d}\right]=\frac{\sqrt{2}}{2}\left(\tilde{d}+d^{\dagger}\right)_{u}^{(2)},}
\end{aligned}
$$

$$
\begin{aligned}
& {\left[\hat{Q}_{u}^{(2)},\left(d^{\dagger} s+s^{\dagger} \tilde{d}\right)_{v}^{(2)}\right]=(-)^{u} \delta_{u,-v}\left(s+s^{\dagger}\right),} \\
& {\left[\hat{Q}_{u}^{(2)},\left(d^{\dagger} \tilde{d}\right)_{v}^{(2)}\right]=\frac{\sqrt{2}}{2}\langle 2 u 2 v \mid 2 u+v\rangle\left(\tilde{d}+d^{\dagger}\right)_{u+v}^{(2)} .}
\end{aligned}
$$

By using the above relations, one can derive

$$
\begin{aligned}
& {\left[\hat{T}_{q}^{(3)}, \hat{H}(\eta, \chi)\right]} \\
& =\frac{3 \sqrt{5} \varepsilon \eta \chi}{28 N}\left\{\sqrt{10}\left[\left(\hat{B}^{(2)} \hat{A}^{(2)}\right)_{q}^{(3)}-\left(\hat{A}^{(2)} \hat{B}^{(2)}\right)_{q}^{(3)}\right]\right. \\
& -2\left[\left(\hat{B}^{(4)} \hat{A}^{(2)}\right)_{q}^{(3)}-\left(\hat{A}^{(2)} \hat{B}^{(4)}\right)_{q}^{(3)}\right]-2 \chi\left[\left(\hat{B}^{(4)} \hat{B}^{(2)}\right)_{q}^{(3)}\right. \\
& \left.\left.-\left(\hat{B}^{(2)} \hat{B}^{(4)}\right)_{q}^{(3)}\right]\right\},
\end{aligned}
$$

where $\hat{A}_{q}^{(2)}=\left(s^{\dagger} \tilde{d}+d^{\dagger} s\right)_{q}^{(2)}$ and $\hat{B}_{q}^{(k)}=\left(d^{\dagger} \tilde{d}\right)_{q}^{(k)}$ with $k=2,4$. Furthermore, by implementing the matrix elements related to the $s$-boson operators under the $\mathrm{U}(6) \supset \mathrm{U}(5) \supset \mathrm{SO}(5) \supset$ $\mathrm{SO}(3)$ basis vectors $\left\{\left|N n_{d} \tau \Delta L\right\rangle\right\}$, where $N, n_{d}$, $\tau$, and $L$ are the quantum number of $\mathrm{U}(6), \mathrm{U}(5), \mathrm{SO}(5)$, and $\mathrm{SO}(3)$, respectively, and $\Delta$ is the additional quantum number to characterize the multiplicity of $L$ in $\tau$, one gets the replacements $s^{\dagger} \rightarrow \sqrt{\hat{n}_{s}+1}$ and $s \rightarrow \sqrt{\hat{n}_{s}}$ with $\hat{n}_{s}=N-\hat{n}_{d}$. Then, in the $n_{d} / N \ll 1$ limit, one can derive that

$$
\begin{aligned}
& {\left[\hat{Q}_{q}^{(2)}, \hat{H}(\eta, \chi)\right]=\frac{\sqrt{2} \varepsilon}{2}(1-2 \eta) \hat{C}_{q}^{(2)}-} \\
& \frac{\sqrt{2} \varepsilon \eta \chi}{8 N}\left[\left(\hat{A}^{(2)} \hat{C}^{(2)}\right)_{q}^{(2)}+\left(\hat{C}^{(2)} \hat{A}^{(2)}\right)_{q}^{(2)}+\right. \\
& \left.2 \hat{B}_{q}^{(2)}+\chi\left(\hat{C}^{(2)} \hat{B}^{(2)}\right)_{q}^{(2)}+\chi\left(\hat{B}^{(2)} \hat{C}^{(2)}\right)_{q}^{(2)}\right],
\end{aligned}
$$

where $\hat{C}_{q}^{(2)}=\left(\tilde{d}+d^{\dagger}\right)_{q}^{(2)}$. In order to make the commutators given in (36) and (37) vanish at the same time, it is uniquely required $(\eta=0.5, \chi=0)$, under which the IBM Hamiltonian just locates at the single triple point mentioned above. The result clearly shows that the Hamiltonian at the triple point is approximately invariant under the $\mathrm{Eu}(5)$ transformations in the $n_{d} / N \ll 1$ limit. It should be noted that the approximation condition $n_{d} / N \ll 1$ is well satisfied for low-lying states generated from the IBM Hamiltonian (27) with $\eta \in[0,0.5]$ and $\chi=0$ in large $N$ cases [35, 38, 39]. In fact, if implementing the matrix elements related to $s$-boson operators under the basis vectors $\left\{\left|N n_{d} \tau \Delta L\right\rangle\right\}$, one can write the Hamiltonian (27) at the triple point $(\eta=0.5, \chi=0)$ as

$$
\begin{aligned}
\hat{H}_{\mathrm{tri}} & =\frac{\varepsilon}{8}\left\{4 \hat{n}_{d}-\frac{1}{N}\left[\hat{n}_{d}\left(N-\hat{n}_{d}+1\right)+\right.\right. \\
& \left(N-\hat{n}_{d}\right)\left(\hat{n}_{d}+5\right)+d^{\dagger} \cdot d^{\dagger} \sqrt{\left(N-\hat{n}_{d}\right)\left(N-\hat{n}_{d}-1\right)}+ \\
& \left.\left.\sqrt{\left(N-\hat{n}_{d}+1\right)\left(N-\hat{n}_{d}+2\right)} \tilde{d} \cdot \tilde{d}\right]\right\} .
\end{aligned}
$$

In the $n_{d} / N \ll 1$ limit, Eq. (38) can be further approximated as [8]

$$
\begin{aligned}
\hat{H}_{\mathrm{tri}} & \simeq \frac{\varepsilon}{4}\left[\hat{n}_{d}-\frac{5}{2}-\frac{1}{2}\left(P_{d}^{\dagger}+P_{d}\right)\right] \\
& =\frac{\varepsilon}{4}\left[\hat{C}_{2}[\operatorname{Eu}(5)]-5\right],
\end{aligned}
$$


which is explicitly given as the Casimir operator of the $\mathrm{Eu}(5)$ up to a constant and a scale factor. It is thus confirmed that the $\mathrm{Eu}(5) \mathrm{DS}$ indeed occurs in the $n_{d} / N \ll 1$ limit at the triple point. Rigorously speaking, the $\mathrm{Eu}(5) \mathrm{DS}$ occurring at the triple point is only almost exact for the ground state in the large- $N$ limit but approximate for the excited states because the condition $n_{d} / N \ll 1$ for the excited states at the triple point becomes weaker with the increasing of the excitation energies. In addition, it should be emphasized that the $\mathrm{Eu}(5) \mathrm{DS}$ is the (approximate) DS associated with a $\beta$-soft potential since the scale potential surface deduced from (29) at the triple point is soft in $\beta$ in contrast to other DSs in the IBM, of which the potential surfaces are all relatively rigid in $\beta$.

\section{POSSIBLE EU(5) CANDIDATES}

Based on the concept of dynamical symmetry, the Hamiltonian with the $\mathrm{Eu}(5) \mathrm{DS}$ can generally be written as

$$
\hat{H}_{\mathrm{Eu}(5)}=a \hat{C}_{2}[\mathrm{Eu}(5)]+b \hat{C}_{2}[\mathrm{SO}(5)]+c \hat{C}_{2}[\mathrm{SO}(3)],
$$

where $a, b$, and $c$ are adjustable parameters, and $\hat{C}_{2}[\mathrm{SO}(5)]$ and $\hat{C}_{2}[\mathrm{SO}(3)]$ are the Casimir operators of $\mathrm{SO}(5)$ and $\mathrm{SO}(3)$ defined as

$$
\begin{aligned}
& \hat{C}_{2}[\operatorname{SO}(5)]=\hat{T}^{3} \cdot \hat{T}^{3}+\hat{T}^{1} \cdot \hat{T}^{1}, \\
& \hat{C}_{2}[\operatorname{SO}(3)]=5 \hat{T}^{1} \cdot \hat{T}^{1},
\end{aligned}
$$

with $\hat{T}^{1}$ and $\hat{T}^{3}$ being those given in (3). One can construct eigenstates of (40) from the $\operatorname{SO}(5)$ basis vectors $\{|\tau \Delta L\rangle \equiv$ $\left.\left|n_{d}=\tau, \tau \Delta L\right\rangle\right\}$, which is well defined in the IBM, since $\mathrm{SO}(5)$ is the subalgebra of the Eu(5) as shown in (6). Specifically, the eigenstates of the Hamiltonian in the $d$-boson system with the seniority $\tau$ and angular momentum $L$ being good quantum numbers can be expressed as

$$
|\xi \tau \Delta L\rangle=\sum_{k=0}^{m} C_{k}^{\xi}\left(\hat{P}_{d}^{\dagger}\right)^{k}|\tau \Delta L\rangle,
$$

where $m+1$ represents the dimension of the Hilbert subspace, and $C_{k}^{\xi}$ is the expansion coefficient with $\xi$ being the additional quantum number to distinguish the states with the same $\tau, \Delta$, and $L$. The expansion coefficients $\left\{C_{k}^{\xi}\right\}$ are determined by the eigen-equation

$$
\hat{H}_{\mathrm{Eu}(5)}|\xi \tau \Delta L\rangle=E_{\tau L}^{\xi}|\xi \tau \Delta L\rangle .
$$

Concretely, one can diagonalize the Hamiltonian (40) in the $m+1$ dimensional Hilbert subspace to get the eigenvalues $E_{\tau L}^{\xi}$ and the corresponding expansion coefficients $\left\{C_{k}^{\xi}\right\}$. Generally, for the IBM Hamiltonian such as that of the O(6) DS, $m=\left[\frac{N-\tau}{2}\right]$ is required, where $[x]$ is the integer part of $x[1]$, and the $s$-boson part $\left|n_{s}\right\rangle$ should also be involved in (43) for the IBM with $n_{s}=N-2 k-\tau$. In the present case, the $s$-boson part is irrelevant, while $m$ should, in principle, be taken as infinite since the dimension of the Hilbert subspace is infinite due to the non-compactness of the $\mathrm{Eu}(5)$ algebra [24]. However, the analysis [21] shows that the dynamical structure of the $\mathrm{Eu}(5) \mathrm{DS}$ may be well kept in the finite- $m$ cases, which indicates that one can diagonalize the Eu(5) Hamiltonian within a finite subspace with sufficient large- $m$ truncation. In our calculation, the parameter $a$ in (40) is reset as $a=\alpha m$ for convenience since the energy levels generated by $\hat{C}_{2}[\mathrm{Eu}(5)]$ may scale with $m^{-1}$ as shown in [21]. In contrast, the energy levels generated by $H_{\text {tri }}$ shown in (38) may scale with $m^{-1 / 3}$ [40]. Besides the diagonalization scheme, it should be mentioned that the eigenstates corresponding to a DS in the IBM may be built through the so called spectrum algebra method. For example, the eigenstates in the O(6) DS can be analytically constructed by acting the generalized boson pairing operators on the $\mathrm{O}(6)$ basis vector [1]. Similarly, as shown in (43), eigenstates of the $\mathrm{Eu}(5) \mathrm{DS}$ are constructed by acting the generalized boson pairing operator $\sum_{k=0}^{m} C_{k}^{\xi}\left(\hat{P}_{d}^{\dagger}\right)^{k}$ on the $\mathrm{SO}(5)$ basis vectors with $m$ being infinite. But the coefficients $C_{k}^{\xi}$ can be only calculated in a numerical way at present.

In the previous work [21], it was shown that the simplest version of $\mathrm{Eu}(5) \mathrm{DS}$ involving only the first term in (40) provides an algebraic description of the E(5) CPS [22], in which the E(5) CPS has been widely confirmed [41-48]. It is thus suggested that the $\mathrm{E}(5)$ nuclei may be the candidate of the $\mathrm{Eu}(5) \mathrm{DS}$ in experiments. Here, we choose ${ }^{108} \mathrm{Pd}$ [49], ${ }^{134} \mathrm{Ba}[50],{ }^{64} \mathrm{Zn}$ [51] and ${ }^{114} \mathrm{Cd}$ [52], which were previously identified as the candidates of the E(5) CPS [41, 42, 46, 47], as examples to show the possible $\mathrm{Eu}(5)$ patterns in experiments. In our calculation, the E2 transition operator is taken as

$$
\hat{T}_{u}=e\left(d^{\dagger}+\tilde{d}\right)_{u}^{(2)}
$$

with the effective boson charge $e$ determined by the corresponding experiment value of $B\left(E 2 ; 2_{1} \rightarrow 0_{1}\right)$ (in W.u.). The low-lying patterns of these $\mathrm{E}(5)$ nuclei and the corresponding results obtained from the $\mathrm{Eu}(5) \mathrm{DS}$ are shown in Fig. 2,5. It can be clearly observed from Fig. 2 that the low-lying spectrum of ${ }^{108} \mathrm{Pd}$ can be well described by the $\mathrm{Eu}(5)$ pattern determined by (40). Particularly, the relative $B(E 2)$ strengths in the $\mathrm{Eu}(5) \mathrm{DS}$ are independent of the parameters, while the data of ${ }^{108} \mathrm{Pd}$ seem to be well reproduced by those of the $\mathrm{Eu}(5) \mathrm{DS}$. In addition, $B\left(E 2 ; 0_{3,2}^{+} \rightarrow 2_{2}^{+}\right)$evidently deviates from $B\left(E 2 ; 0_{\tau}^{+} \rightarrow 2_{2}^{+}\right)$in the $\mathrm{Eu}(5) \mathrm{DS}$, which indicates that the $0_{\xi}^{+}$and $0_{\tau}^{+}$components may be mixing in the excited $0^{+}$ states of ${ }^{108} \mathrm{Pd}$. As shown in Fig. 3,5, the low-lying pattern of ${ }^{134} \mathrm{Ba},{ }^{64} \mathrm{Zn}$, and ${ }^{114} \mathrm{Cd}$ can also be globally reproduced well in the $\mathrm{Eu}(5) \mathrm{DS}$, which further confirms that the $\mathrm{Eu}(5)$ DS emerges in these nuclei. Meanwhile, deviations from the experimental results still exist. For example, $B\left(E 2 ; 3_{1}^{+} \rightarrow 2_{2}^{+}\right)$ calculated from the $\mathrm{Eu}(5) \mathrm{DS}$ description seems too large, and the ordering of the first two excited $0^{+}$levels in ${ }^{134} \mathrm{Ba}$ and ${ }^{64} \mathrm{Zn}$ is altered in the $\mathrm{Eu}(5) \mathrm{DS}$ as show in Figs. 3 and 4 which show that the Eu(5) DS is still an approximate symmetry. As analyzed in [53], the order of the first two excited $0^{+}$states can be altered by using a $\gamma$-independent displaced 

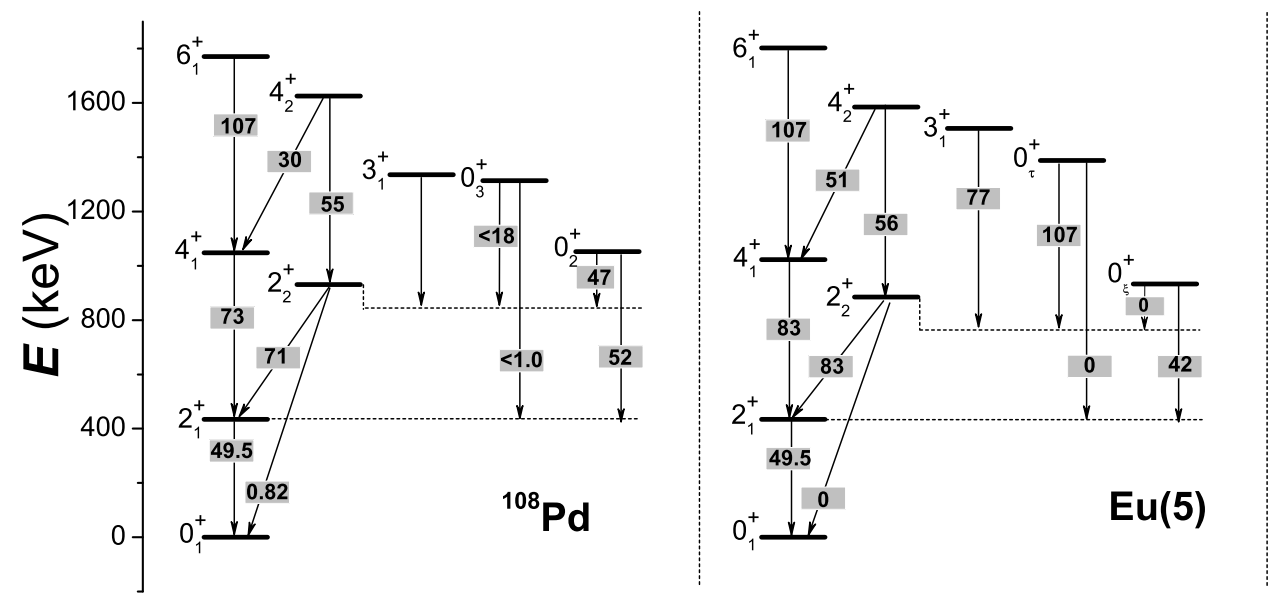

FIG. 2: (Color online) The low-lying structure of ${ }^{108} \mathrm{Pd}$ [49] and the results calculated for the Eu(5) Hamiltonian 40] with the $m=100$ truncation, where $0_{\tau}^{+}$and $0_{\xi}^{+}$represent the excited $0^{+}$state with $\tau=3$ in the $\xi=1$ family and that with $\tau=0$ in the $\xi=2$ family, respectively, as those in the E(5) CPS [22]. The parameters involved in the Eu(5) Hamiltonian are set as $\alpha=49.4 \mathrm{keV}, b=14.8 \mathrm{keV}$, and $c=9.9 \mathrm{keV}$.
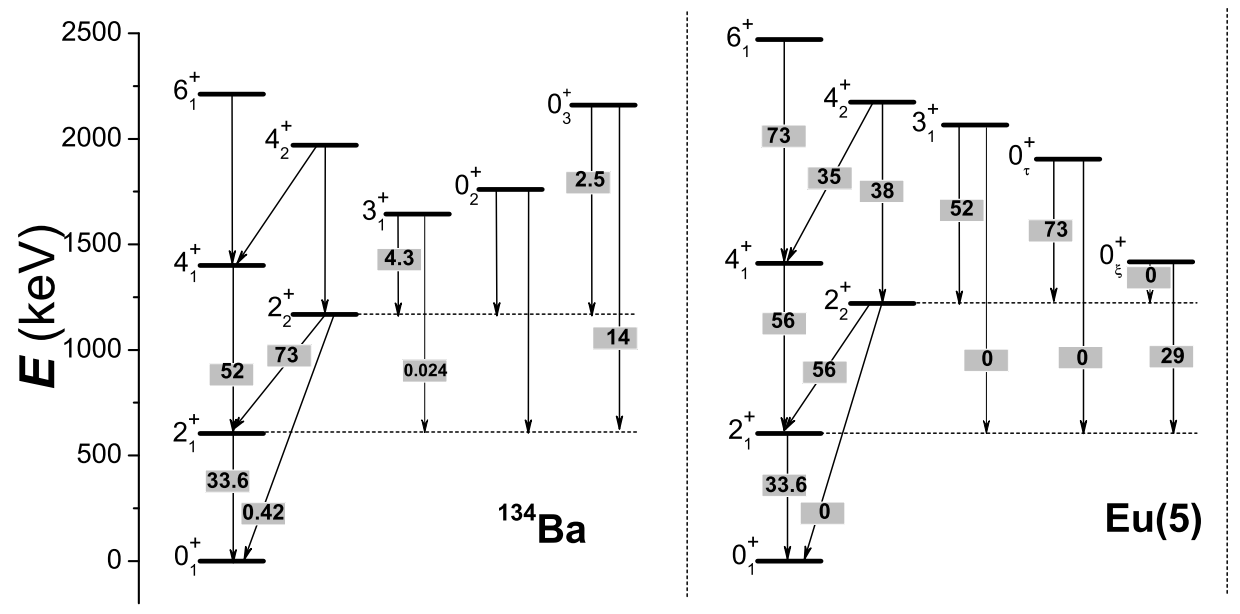

FIG. 3: (Color online) The same as in Fig. 2] but for ${ }^{134} \mathrm{Ba}[50]$ and the corresponding Eu(5) pattern, for which the parameters are set as $\alpha=74.9 \mathrm{keV}, b=11.2 \mathrm{keV}$ and $c=13.5 \mathrm{keV}$.

infinite well $\beta$ potential in the Bohr Hamiltonian in contrast to the one used in the E(5) CPS. It would be very interesting to investigate whether such an improvement in the E(5) CPS will be achieved in the $\mathrm{Eu}(5) \mathrm{DS}$, which may be discussed elsewhere.

In comparison of the $\mathrm{Eu}(5) \mathrm{DS}$ results with those of the $\mathrm{E}(5)$ and the IBM consistent-Q Hamiltonian at the triple point, some typical level energies and $B(E 2)$ ratios calculated from these models in comparison with the corresponding experimental data are shown in Table \ As clearly shown in Table \. the results of these models are similar and accord with the experimental data with quantitative differences. In addition, one may notice from Table \ that the $B(E 2)$ ratios obtained from the E(5) CPS are almost the same as those calculated from the Eu(5) DS with $m=100$ up to the second decimal place. Actually, the minor differences at the second decimal place in the $B(E 2)$ ratios shown in Table $\llbracket$ can also be removed if these quantities are calculated with larger $m$ truncation, which indicates that the $\mathrm{Eu}(5)$ Hamiltonian (40) is just an algebraic equivalent description of the $\mathrm{E}(5)$ CPS with $\mathrm{SO}(5)$ and $\mathrm{SO}(3)$ 

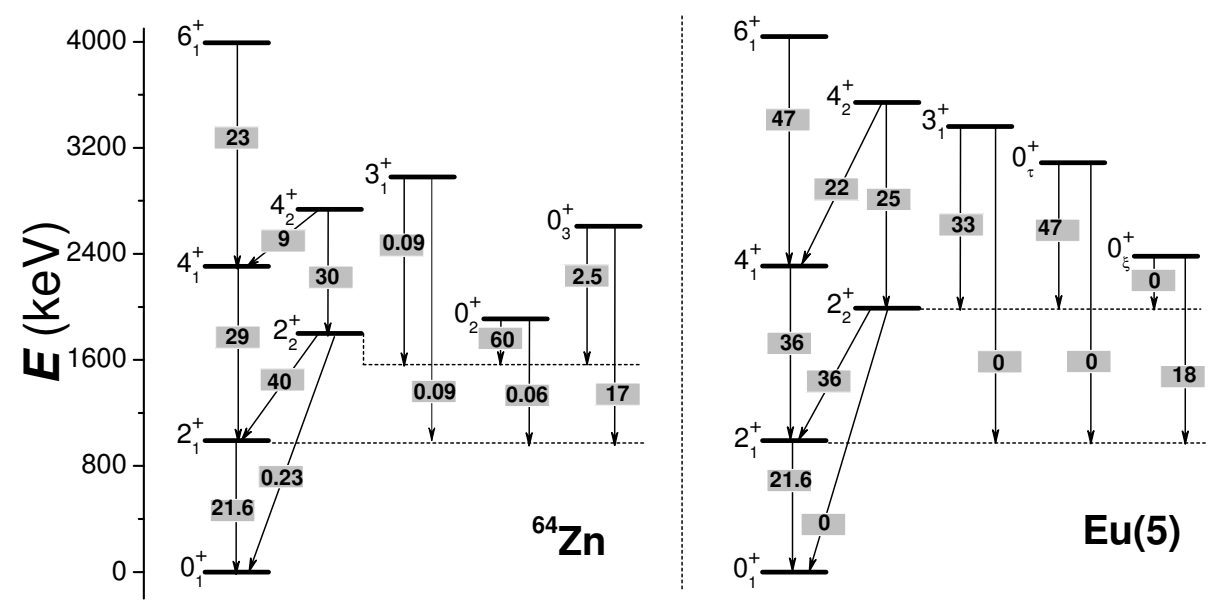

FIG. 4: (Color online) The same as in Fig. 2 but for ${ }^{64} \mathrm{Zn}$ [51] and the corresponding Eu(5) pattern, for which the parameters are set as $\alpha=126 \mathrm{keV}, b=12.6 \mathrm{keV}$ and $c=22.7 \mathrm{keV}$.
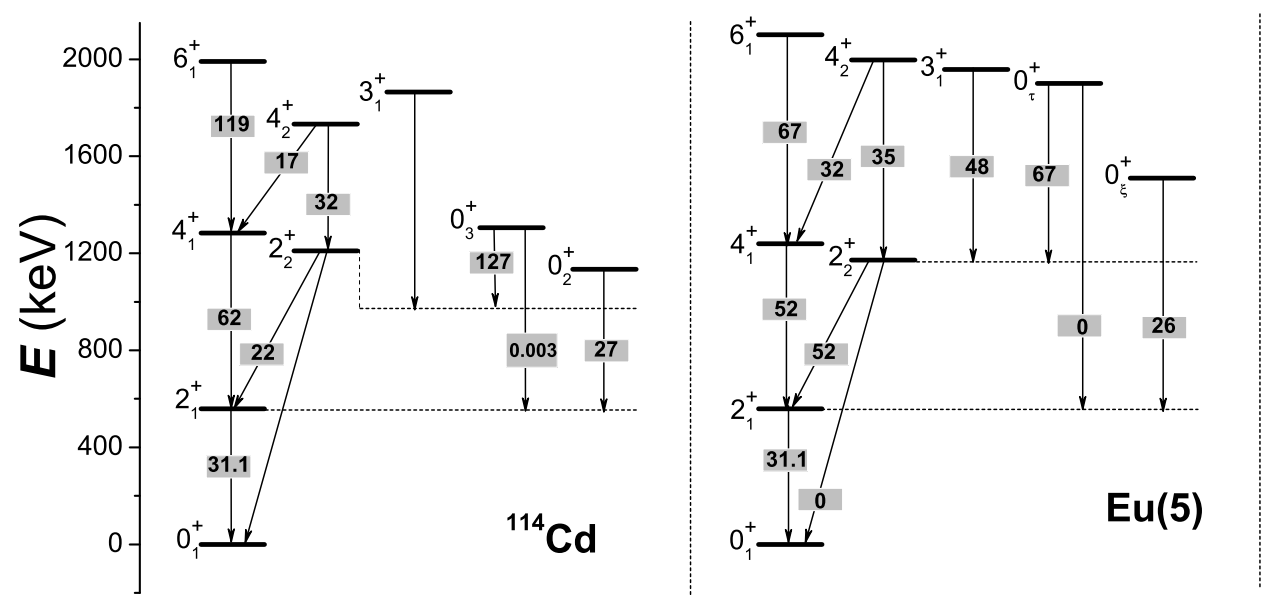

FIG. 5: (Color online) The same as in Fig. 2] but for ${ }^{114} \mathrm{Cd}$ [52] and the corresponding Eu(5) pattern, for which the parameters are set as $\alpha=79.9 \mathrm{keV}, b=4.8 \mathrm{keV}$ and $c=4.8 \mathrm{keV}$.

invariants being involved, with which an intimate relation between the E(5) CPS and the IBM beyond the mean-field approximation [54-56] is thus revealed. It should be mentioned that one can also eliminate the differences of the energy ratios in the $\mathrm{Eu}(5) \mathrm{DS}$ from the $\mathrm{E}(5)$ description shown in Table!by adding a linear combination of the $\mathrm{SO}(5)$ and $\mathrm{SO}(3)$ Casimir operators in the E(5) CPS Hamiltonian [22, 23].

\section{SUMMARY}

In summary, the boson realization of the $\mathrm{Eu}(5)$ algebras has been presented. Based on which the relation between the $\mathrm{Eu}(5)$ dynamical symmetry description and the IBM is discussed. Specifically, it is shown that the Eu(5) dynamical symmetry may emerge at the triple point of the IBM phase diagram in the $n_{d} / N \ll 1$ limit, which thus provides an alternative insight into the dynamical structure at/around this isolated point and the experimental data associated with it. On the other hand, this work also shows that the results of the E(5) CPS can be realized in a fully DS way within the algebraic frame, which thus reveals a more intimate relationship 
TABLE I: Typical energy and $B(E 2)$ ratios of ${ }^{108} \mathrm{Pd}$ [49], ${ }^{134} \mathrm{Ba}$ [50], ${ }^{64} \mathrm{Zn} \mathrm{[51],} \mathrm{and}{ }^{114} \mathrm{Cd}[52]$ calculated from the Eu(5) DS extracted from Fig. 25 the IBM at the triple point with the boson number $N$ taken as the number of valence nucleon (or hole) pairs for each nucleus, and the E(5) CPS [22] in comparison with the corresponding experimental data.

\begin{tabular}{cccccc}
\hline \hline & $\frac{E\left(4_{1}^{+}\right)}{E\left(2_{1}^{+}\right)} \frac{E\left(2_{2}^{+}\right)}{E\left(4_{1}^{+}\right)} \frac{E\left(0_{\xi}^{+}\right)}{E\left(2_{1}^{+}\right)} \frac{B\left(E 2 ; 4_{1}^{+} \rightarrow 2_{1}^{+}\right)}{B\left(E 2 ; 2_{1}^{+} \rightarrow 0_{1}^{+}\right)}$ & $\frac{B\left(E 2 ; 0_{2}^{+} \rightarrow 2_{1}^{+}\right)}{B\left(E 2 ; 2_{1}^{+} \rightarrow 0_{1}^{+}\right)}$ \\
\hline${ }^{108} \mathrm{Pd}$ & 2.42 & 0.89 & 2.43 & 1.48 & 1.05 \\
$\mathrm{Eu}(5)$ & 2.36 & 0.86 & 2.15 & 1.67 & 0.85 \\
$\operatorname{Tri}(N=8)$ & 2.14 & 1.00 & 3.00 & 1.55 & 0.81 \\
$\mathrm{E}(5)$ & 2.20 & 1.00 & 3.03 & 1.68 & 0.86 \\
\hline${ }^{134} \mathrm{Ba}$ & 2.32 & 0.83 & 3.57 & 1.55 & 0.42 \\
$\mathrm{Eu}(5)$ & 2.33 & 0.87 & 2.34 & 1.67 & 0.85 \\
$\operatorname{Tri}(N=5)$ & 2.15 & 1.00 & 3.18 & 1.41 & 0.66 \\
$\mathrm{E}(5)$ & 2.20 & 1.00 & 3.03 & 1.68 & 0.86 \\
\hline${ }^{64} \mathrm{Zn}$ & 2.33 & 0.78 & 2.63 & 1.34 & 0.79 \\
$\mathrm{Eu}(5)$ & 2.33 & 0.86 & 2.40 & 1.67 & 0.85 \\
$\operatorname{Tr}(N=4)$ & 2.16 & 1.00 & 3.29 & 1.32 & 0.58 \\
$\mathrm{E}(5)$ & 2.20 & 1.00 & 3.03 & 1.68 & 0.86 \\
\hline${ }^{114} \mathrm{Cd}$ & 2.30 & 0.94 & 2.03 & 1.99 & 0.87 \\
$\mathrm{Eu}(5)$ & 2.22 & 0.95 & 2.70 & 1.67 & 0.85 \\
$\operatorname{Tr}(N=9)$ & 2.14 & 1.00 & 2.96 & 1.57 & 0.84 \\
$\mathrm{E}(5)$ & 2.20 & 1.00 & 3.03 & 1.68 & 0.86 \\
\hline \hline
\end{tabular}

between the E(5) CPS and the IBM beyond the mean-field approximation. Finally, a preliminary examination of the $\mathrm{Eu}(5)$ DS in ${ }^{108} \mathrm{Pd},{ }^{134} \mathrm{Ba},{ }^{64} \mathrm{Zn}$, and ${ }^{114} \mathrm{Cd}$ is made. The results indicate that the low-lying dynamics of these nuclei are indeed dominated by the Eu(5) DS.

Supports from U.S. National Science Foundation (OCI0904874), Southeastern Universities Research Association, the Natural Science Foundation of China (11375005, 11005056, 11175078, 11175004, 11435001 and 11475091), and the LSU-LNNU joint research program (9961) are acknowledged. YXL thanks also the support from the National Key Basic Research Program of China under Contract No. G2013CB834400.

[1] F. Iachello, and A. Arima, The Interacting Boson Model (Cambridge University, Cambridge, England, 1987).

[2] F. Iachello, and R. D. Levine, Algebraic Theory of Molecules (Oxford University, Oxford, UK 1995).

[3] H. Yépez-Martínez, J. Cseh, and P. O. Hess, Phys. Rev. C 74, 024319 (2006).

[4] J. Jolie, R. F. Casten, P. von Brentano, and V. Werner, Phys. Rev. Lett. 87, 162501 (2001).

[5] A. Leviatan, Phys. Rev. Lett. 77, 818 (1996).

[6] A. Leviatan, Phys. Rev. Lett. 98, 242502 (2007).

[7] J. E. Garcìa-Ramos, A. Leviatan, and P. Van Isacker, Phys. Rev. Lett. 102, 112502 (2009).
[8] D. J. Rowe, Phys. Rev. Lett. 93, 122502 (2004).

[9] D. J. Rowe, P. S. Turner, and G. Rosensteel, Phys. Rev. Lett. 93, 232502 (2004).

[10] D. J. Rowe, Nucl. Phys. A 745, 47 (2004).

[11] D. Bonatsos, E. A. McCutchan, and R. F. Casten, Phys. Rev. Lett. 104, 022502 (2010).

[12] D. Bonatsos, S. Karampagia, and R. F. Casten, Phys. Rev. C 83, 054313 (2011).

[13] Y. Alhassid and N. Whelan, Phys. Rev. Lett. 67, 816 (1991).

[14] J. Jolie, et al., Phys. Rev. Lett. 93, 132501 (2004).

[15] C. Kremer, et al., Phys. Rev. C 89, 041302(R) (2014).

[16] P. Cejnar, J. Jolie, and R. F. Casten, Rev. Mod. Phys. 82, 2155 (2010).

[17] P. Cejnar, and J. Jolie, Prog. Part. Nucl. Phys. 62, 210 (2009).

[18] R. F. Casten and E. A. McCutchan, J. Phys. G 34, R285 (2007).

[19] J. Jolie, P. Cejnar, R. F. Casten, S. Heinze, A. Linnemann, and V. Werner, Phys. Rev. Lett. 89, 182502 (2002).

[20] D. Warner, Nature 42, 614 (2002).

[21] Y. Zhang, Y. X. Liu, F. Pan, Y. Sun, and J. P. Draayer, Phys. Lett. B 732, 55 (2014).

[22] F. Iachello, Phys. Rev. Lett. 85, 3580 (2000).

[23] M. A. Caprio, and F. Iachello, Nucl. Phys. A 781, 26 (2007).

[24] B. G. Wybourne, Classical Groups for Physicists (Wiley, New York, 1974).

[25] P. Feinsilver, Acta Appl. Math. 43, 289 (1996).

[26] D. Bonatsos, E. A. McCutchan, and R. F. Casten, Phys. Rev. Lett. 101, 022501 (2008).

[27] H. Ui, Prog. Theor. Phys. 44, 153 (1970).

[28] D. J. Rowe, Nucl. Phys. A 735, 372 (2004).

[29] D. J. Rowe and P. S. Turner, Nucl. Phys. A 753, 94 (2005).

[30] D. J. Rowe, J. Phys. A 38, 10181 (2005).

[31] D. J. Rowe, T. A. Welsh, and M. A. Caprio, Phys. Rev. C 79, 054304 (2009).

[32] F. Pan and J. P. Draayer, Nucl. Phys. A 636, 156 (1998).

[33] F. Pan, X. Zhang, and J. P. Draayer, J. Phys. A 35, 7173 (2002).

[34] D. D. Warner, and R. F. Casten, Phys. Rev. C 28, 1798 (1983).

[35] F. Iachello and N. V. Zamfir, Phys. Rev. Lett. 92, 212501 (2004).

[36] F. Iachello, N. V. Zamfir, and R. F. Casten Phys. Rev. Lett. 81, 1191 (1998).

[37] A. R. Edmonds, Angular Momentum in Quantum Mechanics (Princeton University Press, Princeton, 1957).

[38] J. M. Arias, J. Dukelsky, and J. E. Garcìa-Ramos, Phys. Rev. Lett. 91, 162502 (2003).

[39] F. Pan, Y. Zhang, and J. P. Draayer, J. Phys. G 31, 1039 (2005).

[40] S. Dusuel, J. Vidal, J. M. Arias, J. Dukelsky, and J. E. GarcìaRamos, Phys. Rev. C 72, 011301(R) (2005).

[41] D. L. Zhang and Y. X. Liu, Phys. Rev. C 65, 057301 (2002).

[42] R. F. Casten and N. V. Zamfir Phys. Rev. Lett. 85, 3584 (2000).

[43] A. Frank, C. E. Alonso, and J. M. Arias, Phys. Rev. C 65, 014301 (2001).

[44] N. V. Zamfir, et al., Phys. Rev. C 65, 044325 (2002).

[45] R. M. Clark, et al., Phys. Rev. C 69, 064322 (2004).

[46] C. Mihai, et al., Phys. Rev. C 75, 044302 (2007).

[47] J. F. Zhang, et al., Chin. Phys. Lett. 20, 1231 (2003).

[48] L. Coquard, et al., Phys. Rev. C 80, 061304(R) (2009)

[49] J. Blachot, Nucl. Data Sheets 81, 599 (1997).

[50] A. A. Sonzogni, Nucl. Data Sheets 103, 1 (2004).

[51] B. Singh, Nucl. Data Sheets 78, 395 (1996).

[52] J. Blachot, Nucl. Data Sheets 97, 593 (2002).

[53] D. Bonatsos, D. Lenis, N. Pietralla, and P. A. Terziev and A. Frank, Phys. Rev. C 74, 044306 (2006).

[54] J. M. Arias, C. E. Alonso, A. Vitturi, J. E. Garcìa-Ramos, J. Dukelsky, and A. Frank, Phys. Rev. C 68, 041302(R) (2003). 
[55] J. E. Garcìa-Ramos and J. M. Arias, Phys. Rev. C 77, 054307

Mech. Astron. 54, 227 (2011). (2008).

[56] Y. Zhang, F. Z. Hou, and Y. X. Liu, and Y. Sun, Sci. China Phys. 